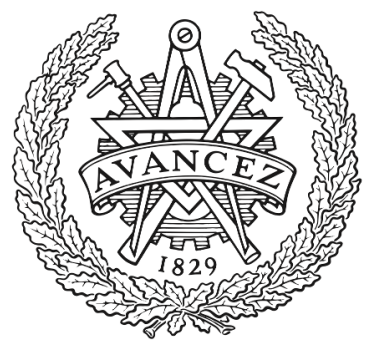

CHALMERS

UNIVERSITY OF TECHNOLOGY

\title{
The Effect of Bending Deformation on Charge Transport and Electron Effective Mass of p-doped GaAs Nanowires
}

Downloaded from: https://research.chalmers.se, 2023-04-26 15:27 UTC

Citation for the original published paper (version of record):

Zeng, L., Kanne, T., Nygard, J. et al (2019). The Effect of Bending Deformation on Charge Transport and Electron Effective Mass of p-doped GaAs Nanowires. Physica Status Solidi - Rapid Research Letetrs, 13(8).

http://dx.doi.org/10.1002/pssr.201900134

N.B. When citing this work, cite the original published paper. 


\section{The Effect of Bending Deformation on Charge Transport and Electron Effective Mass of p-doped GaAs Nanowires}

Lunjie Zeng, Thomas Kanne, Jesper Nygård, Peter Krogstrup, Wolfgang Jäger, and Eva Olsson $^{*}$

Dr. L.J. Zeng, Prof. W. Jäger, Prof. E. Olsson

Department of Physics, Chalmers University of Technology, 41296, Gothenburg, Sweden

Email: eva.olsson@,chalmers.se

T. Kanne, Prof. J. Nygård, Prof. P. Krogstrup

Center for Quantum Devices, Nano-Science Center, Niels Bohr Institute, University of Copenhagen, 2100 Copenhagen, Denmark

Prof. W. Jäger

Institute for Materials Science, Christian-Albrechts-University Kiel, 24143, Kiel, Germany

\section{Keywords}

strain engineering, bending deformation, GaAs nanowire, charge transport, band structure

\section{Abstract}

The crystal and electronic structure of semiconductor nanowire systems have shown sensitive response to mechanical strain, enabling novel and improved electrical and optoelectrical properties in nanowires by strain engineering. Here, the response of current-voltage (I-V) characteristics and band structure of individual p-doped GaAs nanowires to bending deformation is studied by in situ electron microscopy combined with theoretical simulations. The I-V characteristics of the nanowire change from linear to nonlinear as bending deformation is applied. The nonlinearity increases with strain. As opposed to the case of uniaxial strain in GaAs, the bending deformation does not give rise to a change in the band gap of GaAs nanowire according to in situ electron energy loss spectroscopy (EELS) measurements. Instead, the response to bending deformation can be explained by strain induced valence band shift, which results in an energy barrier for charge carrier transport along the nanowire. Moreover, the electron effective mass decreases as the strain changes 
from compressive to tensile across the GaAs nanowire in the bent region. Results from this study shed light on the complex interplay between lattice strain, band structure and charge transport in semiconductor nanomaterials. 


\section{WILEY-VCH}

\section{Main text}

Strain engineering is an effective way of modifying the crystal and electronic band structure of semiconductor nanowires and enhancing the performance of nanowire-based electronic and optoelectronic devices. ${ }^{[1-9]}$ Nanowires often show large yield strength and can withstand large strains without fracture compared to their bulk counterparts. The mechanical flexibility is a result of their large surface-volume ratio and small size ${ }^{[10,11]}$. The band structure of the semiconductor nanowires is also sensitive to mechanical strain. ${ }^{[6,7,12]}$ Strain induced changes in electrical and optical properties have been observed in various semiconductor nanowires. For example, giant piezoresistance effects have been found in Si nanowires and attributed to the enhancement of carrier mobility induced by the applied compressive stress. ${ }^{[13]}$ Band gap modification resulting in a change in electronic and optical properties of $\mathrm{ZnO}$ nanowires has been achieved by uniaxial strains. ${ }^{[6,12]}$ It has also been shown that uniaxial strain can result in a change from direct to indirect band gap in wurtzite GaAs nanowires. ${ }^{[7]}$ The resistivity of InAs nanowires has shown sensitive response to uniaxial tensile strain. ${ }^{[11,14]}$ In addition, tensile strain has been used to change the band gap of Ge nanowires from indirect to direct. ${ }^{[15]}$ A strong gradient strain, e.g. due to bending deformation, also has a significant impact on the electrical and optical properties of semiconductor nanowires. Moreover, novel functionalities have been found in bent semiconductor nanowires. The inhomogeneous strain fields caused by an externally applied stress in Ge nanowires have been used to enable a strained photovoltaic function within single undoped nanowires. ${ }^{[16]}$ A piezoresistance effect in Si nanowires has been induced by elastic bending deformation. ${ }^{[17]}$ The strain gradient induced by the bending deformation has been used to drift excitons in $\mathrm{ZnO}$ nanowires and tune the exciton dynamics. ${ }^{[18,19]}$

GaAs nanowires are promising components for nanoscale optical and electronic devices, such as solar cells, photodetectors and logic transistors. Those applications of GaAs nanowires 


\section{WILEY-VCH}

originate from their unique band structure characteristics, such as a direct band gap with the energy within the visible light interval, small effective electron mass and high charge carrier mobility. ${ }^{[20-22]}$ The band structure of GaAs nanowires shows sensitive response to externally applied uniaxial stress, resulting in the prospect of development of a new generation of electronic and photonic devices based on GaAs nanowires by utilization of the strain as additional degree of freedom. Uniaxial strain has been shown to cause changes of the band gap value of zinc blende GaAs nanowires. ${ }^{[8]}$ The band gap of wurtzite GaAs nanowire can even be modified from a direct band gap to psudo-indirect band gap under the influence of a small compressive strain. ${ }^{[7]}$ In addition, detailed strain distribution analysis of bending deformation in GaAs nanowire has been carried out using X-ray and electron microscopy techniques. ${ }^{[23,24]}$ However, the effects of the elastic bending deformation on the transport properties and the electronic structure of GaAs nanowires have not yet been investigated.

In this work, we exploit the advantages of performing electrical transport measurements in the transmission electron microscope (TEM). This allows us to directly correlate the local bending deformation of individual GaAs nanowires to changes in properties and electronic structure. An in situ TEM setup with a scanning tunneling microscope (STM) nanoprobe was used to continuously apply bending strain on individual GaAs nanowires, measure the I-V characteristics of the nanowires and also estimate the strain level in the nanowires. ${ }^{[25]}$ The band gap of the strained nanowires was simultaneously measured using electron energy loss spectroscopy (EELS). Tight-binding simulations were used to reveal the modification of the valence band by mechanical strain. Furthermore, the variation in the effective mass of electrons in the nanowires was investigated by studying the change in bulk plasmon energy determined by EELS. The possibility of strain engineering of the electric transport properties and band structure of GaAs nanowires with bending deformation may provide a new venue for the optimization of GaAs nanowire based devices. 
The in situ TEM sample preparation procedure and the in situ TEM set up are shown in Figure 1. Special attention was paid to establishing Ohmic and low resistance contacts. A pdoped GaAs nanowire was first mechanically removed from the growth substrate by the micromanipulator in focus ion beam (FIB) - scanning electron microscope (SEM) and attached to the manipulator by Van der Waals force between the nanowire and the manipulator (Figure 1a). The nanowire was thereafter transferred to a $\mathrm{Cu}$ TEM grid. One end of the nanowire was fixed to the TEM grid using electron beam induced deposition (EBID) of Pt. The $\mathrm{Cu}$ TEM grid with nanowire on it was mounted in the in situ holder. The moveable nanoprobe $(\mathrm{Au})$ in the TEM holder was used to contact the tip of the nanowire during in situ straining and electrical transport properties measurements (Figure $1 \mathrm{~b}$ and c). Since a twoprobe electrical measurement set up was used in our in situ TEM experiment, it is important to minimize the effect of electrical contacts on the in situ transport property measurements. When the nanoprobe in the TEM holder directly contacts the nanowire, we find that the contact area between the nanoprobe and the nanowire often changes when the probe moves and applies stress on the nanowire. Such a change in contact condition may result in changes in the contact resistance. In addition, the Schottky barrier between the nanoprobe $(\mathrm{Au})$ and GaAs nanowire can be affected by the force applied between the probe and the nanowire. The changes in Schottky barrier properties (barrier height and width) due to strain at the contact may significantly alter the I-V characteristics and deter the investigation of the effect of strain on the intrinsic electrical transport properties of the nanowires. To minimize the effect of the changes in contact resistance and the Schottky barrier between the nanoprobe and the nanowire ( $\mathrm{Au} / \mathrm{GaAs}$ junction), we welded a small $\mathrm{Au}$ contact piece at the tip of the nanowire in FIB-SEM, followed by controlled Joule heating of the contacts in TEM. With this approach, the contact condition between the $\mathrm{Au}$ nanoprobe and the Au contact piece at the nanowire tip may still change during in situ straining. But the effect of this contact on the I-V 


\section{WILEY-VCH}

characteristics is minor due to the fact that it is a metal/metal contact with a small contact resistance. The contact resistance between the Au contact piece and GaAs nanowire will not change significantly during the in situ bending process since this contact is fixed. Moreover, the addition of the Au contact piece at the tip of the nanowire and controlled Joule heating of the electric contacts (with a current of $\sim 500 \mathrm{nA}$ ) greatly diminished the Schottky barrier. Before optimizing the contact with the aforementioned process, the I-V characteristics of the nanowire are dominated by the nanoscale Schottky barrier between the nanoprobe and the nanowire (Figure 1d) and shows nonlinear and asymmetric behavior. ${ }^{[26]}$ After the optimization process, the resulting I-V curve appears linear and nearly shows Ohmic behavior as expected for a highly p-doped GaAs nanowire (Figure 1d). ${ }^{[22]}$ From the TEM bright field (BF) images (e.g., Figure 2a and Figure 3a), the length and diameter of the nanowire were measured. The resistivity of the GaAs nanowire was estimated to be $\sim 2 \times 10^{-3} \Omega \cdot \mathrm{m}$, which is in good agreement with that measured using a standard 4-probe method on the nanowires grown under the same conditions. ${ }^{[2]}$ It further indicates that after the contact optimization process, the contact resistance in the circuit is insignificant compared to the resistance of the nanowire. $^{[22]}$

The length of the nanowires we studied is in the range of $10-20 \mu \mathrm{m}$ and their diameter is around $250 \mathrm{~nm}$. The nanowires have zinc blende crystal structure with twin domains distributing along the nanowires (Figure 2a). The average number density of twin domains along the nanowire growth direction is around $6 / \mu \mathrm{m}$. High resolution scanning transmission electron microscopy (STEM) annular dark field (ADF) imaging shows the zinc blende crystal structure of a nanowire, with twin boundaries perpendicular to the nanowire growth direction ([111] direction), as shown in Figure 2d. It has been shown previously that such twin domains and boundaries have insignificant effects on the electrical transport properties of GaAs nanowires at room temperature. ${ }^{[27,28]}$ 


\section{WILEY-VCH}

Mechanical stress was applied on the GaAs nanowire by using a nanoprobe in the in situ TEM holder. The probe first contacted the Au contact piece at the tip of the nanowire. Then the probe moved towards the TEM grid and pushed the nanowire, resulting in bending deformation (Figure 3a-d). As the nanowire was bent gradually, I-V curves were acquired by sweeping the applied voltage from $-1 \mathrm{~V}$ to $1 \mathrm{~V}$ and measuring the current through the nanowire

(Figure $3 \mathrm{e}$ and $\mathrm{f}$ ). The sweeping rate of $\mathrm{I}-\mathrm{V}$ measurement was $1000 \mathrm{mV} / \mathrm{s}$. We did not observe hysteresis in I-V curves. The strain $\varepsilon$ in the nanowire was determined from the dimensional parameters of the bent nanowire in TEM images (Figure 3e) using the relation:

$$
\varepsilon=\frac{y}{R}
$$

where $y$ is the projected distance of regions strained in tension $(y>0)$ or compression $(y<0)$ from the neutral axis of a nanowire of radius $r . R$ is the radius of curvature of the bent part of the wire. On the outer perimeter side of the bent wire, the strain is tensile. On the inner side, the wire is in compression. The degree of bending is characterized by the maximum strain $\varepsilon_{\max }=r / R$ in the nanowire. ${ }^{[29]}$

The I-V characteristics of GaAs nanowires were measured at continuously increasing elastic deformation, i.e. while the nanowire was deformed continuously in the TEM. Representative TEM images of a nanowire with four different levels of strain are shown in Figure 3a-d. The corresponding I-V curves are shown in Figure 3f.

Figure $3 \mathrm{f}$ shows that the I-V curve is linear when there is no stress applied on the nanowire. The I-V curve becomes nonlinear when the amount of maximum strain $\left(\varepsilon_{\max }\right)$ is around $0.5 \%$. The degree of nonlinearity of the I-V curve gradually increases with increasing bending deformation, as shown for the range of $\varepsilon_{\max }$ up to around $3 \%$. It is also worth noting here that the microstructure of the nanowire is identical to the initial state after external stress release as 


\section{WILEY-VCH}

shown in Fig 2a-c. This result confirms that the nanowire remain within the elastic deformation regime in the present measurements. This is consistent with our finding that when the stress is released, the I-V characteristics of the nanowire also coincide with those acquired in the beginning of the experiment, when there is no external stress on the nanowire. The elastic limit of the nanowire used in this study is estimated to be around $\varepsilon_{\max }=5 \%$ strain $\left(\varepsilon_{\max }\right)$. When the strain exceeded $5 \%$, the nanowire experienced a permanent change in morphology and did not recover its original shape, or it even broke. We have performed in situ TEM measurements on 5 nanowires from the same growth substrate. They show the same behavior in $\mathrm{I}-\mathrm{V}$ characteristics due to bending deformation. $\mathrm{I}-\mathrm{V}$ curve changes from linear to nonlinear when stress is applied. The nonlinearity increases with bending deformation. Results from another nanowire are shown in the Supporting information (S3).

The changing nonlinearity in the $\mathrm{I}-\mathrm{V}$ characteristics indicates the development of an energy barrier for charge carriers along the nanowire length direction due to the bending deformation. In situ STEM-EELS measurements were carried out on bent GaAs nanowires to investigate the effect of bending strain on the electronic structure of the nanowires. Figure 4 shows results from measurements that were performed in the bent region of the GaAs nanowire for which the maximum strain was about 3\%. Figure $4 \mathrm{~b}$ shows a valence EELS (VEELS) lineprofile acquired across the nanowire, starting from the compression and moving to the tension side, in the energy loss range from $\sim 1 \mathrm{eV}$ to $\sim 17 \mathrm{eV}$. The step size between each EELS acquisition was $\sim 10 \mathrm{~nm}$. A band gap of a semiconductor or insulator will give rise to an onset edge superposed on the tail of the zero loss peak in the valence EELS signal. This onset signal between 1 to $2 \mathrm{eV}$ is indicated in the spectra in Figure 4b (also see Figure S1, Supporting Information). Another prominent feature in the spectra in Figure $4 \mathrm{~b}$ is the bulk plasmon peak around $16 \mathrm{eV}$, resulting from the collective movement of valence electrons excited by incident electron beam in TEM. For band gap analysis, the EELS line-profile in the energy 


\section{WILEY-VCH}

loss range between $\sim 1 \mathrm{eV}$ and $5 \mathrm{eV}$ is shown in Figure 4c. The band gap onset between $\sim 1.2$ $\mathrm{eV}$ and $2 \mathrm{eV}$ can be clearly seen in every spectrum. The fine features after $2 \mathrm{eV}$ correspond to interband transitions close to the Fermi level. The energy at the midpoint of the onset edge in the valence EEL spectrum was taken as the band gap value. For example, in the first spectrum from the top in Figure 4c, the midpoint of the onset edge and thus the band gap of the GaAs nanowire is estimated to be $\sim 1.51 \mathrm{eV}$, which is consistent with the band gap values of GaAs

bulk and nanowires reported before. ${ }^{[30]}$ Moving from the region with highest compressive strain (subsequent EEL spectra in the upper part of Figure 4c) to the center of the nanowire, the band gap onset in the spectrum becomes less and less steep, and the total intensity of the spectrum between $\sim 1 \mathrm{eV}$ and $\sim 5 \mathrm{eV}$ decreases. The shape of the band gap onset and the intensity of the spectrum show the opposite trend from the center of the nanowire to the region with highest tensile strain. Such a change in energy loss signals is mainly due to the change in material thickness along the incident beam direction because of the hexagonal cross-section of the nanowire. Nonetheless, the band gap onset can be identified in all the spectra across the nanowire from the compression to the tension side (Figure 4c, dashed line marker). In the bent region, the measured band gap values show little variation at different locations across the nanowire. Thus, our STEM-EELS measurements show that bending deformation does not significantly change the band gap of the GaAs nanowires. This observation is also confirmed by our in situ scanning electron microscope (SEM) cathodoluminescence (CL) measurement on the GaAs nanowires (Figure S2, Supporting Information). The position of the CL peak at around $860 \mathrm{~nm}$, which corresponds to the band gap energy of GaAs, does not detectably shift across the bent nanowire.

Mechanical strain can modify the energy bands of GaAs, especially the top of the valence band, which is critical for hole transport in p-doped GaAs nanowires. To investigate the effect of strain on the valence bands, an $\mathrm{sp} 3 * \mathrm{~d} 5$ tight-binding simulation was performed. ${ }^{[31]}$ In the 


\section{WILEY-VCH}

simulation, only the uniaxial strain along the [111] direction, which is the nanowire length direction, was considered. In general, mechanical strain can split and shift the energy bands in GaAs. The top of the valence band of GaAs consists of split-off, heavy-hole and light-hole bands. When GaAs is unstrained, the two hole bands are degenerate and the energy difference to the split-off band is around $0.3 \mathrm{eV}$ (Figure 5a). The valence band maximum is at the position of the heavy- and light-hole bands. As shown in Figure 5a, mechanical stain, either tensile or compressive strain, can split and shift the two hole bands. Under increasing tensile strain along the nanowire length direction, the heavy-hole band will shift upwards in energy, while the light-hole band will move downwards along the energy axis. As a result, the heavyhole band forms the valence band maximum, which is thus shifted upwards in energy compared to that when the nanowire is unstrained. Conversely, when there is increasing compressive strain in the GaAs nanowire along the length direction, the light-hole band will shift upwards and the heavy-hole band will move downwards in energy. In this case, the valence band maximum becomes the light-hole band, which is also shifted upwards.

Over the whole range of (tensile and compressive) strain, the heavy-hole band shifts linearly with mechanical strain while the light-hole band shows a more parabolic behavior due to the coupling with the split-off band. As a result, both the uniaxial tensile and compressive strains lift the valence band maximum. These results are in accordance with calculations performed using the k.p method. ${ }^{[8]}$ When the p-doped GaAs nanowire was bent as shown in Figure 4a, the most strained region was close to the middle of the nanowire. In the bent region, the valence band maximum energy moves up due to strain (both compressive and tensile) compared to the unbent areas at the two ends of the nanowire. Thus, the shift of the valence band energy results in an energy barrier for holes to travel from one end of the nanowire to the other end (Figure 5b). This energy barrier increases with strain and we anticipate that it leads to the nonlinearity behavior observed in the I-V characteristics shown in Figure 3. 


\section{WILEY-VCH}

In addition, the effect of strain on the effective mass of valence electrons was revealed by STEM-EELS. The most prominent feature in the VEELS is the bulk plasmon peak, which is due to the collective movement of loosely bound electrons excited by the incident highenergy electron beam. ${ }^{[32]}$ In the Drude model, the energy of a plasmon excited in a free electron gas is

$$
E_{p}=\hbar \omega_{p}=\hbar\left[n e^{2} /\left(\varepsilon_{0} m_{0}\right)\right]^{1 / 2}=\hbar e\left[\frac{N}{\varepsilon_{0} m_{0}}\right]^{1 / 2}(1 / V)^{1 / 2} \propto(1 / V)^{1 / 2}
$$

where $n$ is the electron density, $e$ is the electron charge, $\varepsilon_{0}$ is the permittivity of free space and $m_{0}$ is the rest mass of electron. The valence electron density is related to the volume of the unit cell by $n=N / V$, where $N$ is the total number of valence electrons in a unit cell and $\mathrm{V}$ is the volume of the unit cell. In this free electron approximation, the maximum of the energy loss function is then described as

$$
E_{\max }=\left[E_{p}^{2}-\left(\Delta E_{p}^{2}\right)\right]^{1 / 2}
$$

where $E_{\max }$ is the plasmon peak position and $\Delta E_{p}$ is the full width at half maximum (FWHM) of the plasmon peak. Equation (3) shows that $E_{\max }$ is shifted to lower energy because of the plasmon damping. It has been shown before that such a model can be used to quantitatively describe bulk plasmon energy not only of free-electron metals but also of semiconductors, including GaAs. ${ }^{[32]}$

To determine the plasmon energy $\left(E_{\mathrm{p}}\right)$, the single scattering distribution (SSD) was obtained by deconvolution of the EELS spectra using the Fourier-log method. ${ }^{[32]}$ We determined the maximum position of the plasmon peak $\left(E_{\max }\right)$ and the full width at half maximum $\left(\Delta E_{\mathrm{p}}\right)$ of the plasmon peak in the SSD of VEELS by fitting the plasmon peak to a Gaussian function. $E_{\mathrm{p}}$ was then obtained from $E_{\max }$ and $\Delta E_{\mathrm{p}}$ according to Equation 2. The plasmon energy $\left(E_{\mathrm{p}}\right)$ was mapped out in the region where the nanowire was bent (Figure 6a). The plasmon energy 


\section{WILEY-VCH}

map clearly shows the 2D distribution of the bulk plasmon energy in the strained region. The value of the plasmon energy $E_{\mathrm{p}}$ changes across the nanowire when changing the strain state from tension to compression. The energy line profiles for the bulk plasmon (Figure 6c) are obtained from the plasmon energy maps (Figures 6a and b). The upper and lower boundaries in the map were excluded from the data analysis because of the largely reduced bulk plasmon signals close to the surface. The line profiles show that the values of the plasmon energy decrease from the regions under compression to the regions under tension (Figure 6c). Such a variation in plasmon energy is qualitatively in agreement with the predictions of a free electron model, for which the changes in the plasmon energy are due to the variations in volume of the unit cell, or in other words, the lattice parameter. In the areas where there is tensile strain, the crystal lattice expands, and the volume of the unit cell $V$ increases. As a result, the value of $E_{\mathrm{p}}$ decreases in these regions. Conversely, compressive strain results in a reduction of $V$ and an increase of $n$ and $E_{\mathrm{p}}$.

STEM-EELS measurements were also performed on the same area of the unstrained nanowire (Figure 6b). The plasmon energy is found to be around $16 \mathrm{eV}$ across the entire nanowire, with only little variation (Figure 6c). Using the average value of the plasmon energy of the unstrained nanowire as the reference value, the expected changes in plasmon energy due to lattice strain were calculated using the free electron model, as shown in Figure $6 \mathrm{c}$. In the calculation, an uniaxial strain along the [111] direction was assumed, and it was assumed that the change in plasmon energy resulted from the change in the volume of the lattice (see Figure S4, Supporting information). It is clear from Fig. 6c that the simulated values deviate from the experimental EELS results. In the simulated results, the plasmon energy continuously decreases from $\sim 16.19 \mathrm{eV}$ to $\sim 15.80 \mathrm{eV}$ when moving the sampling electron beam from regions of compression to regions of tension, showing an almost linear change with beam positions. In contrast, the measured plasmon energy decreases from $\sim 16.21 \mathrm{eV}$ to $\sim$ 


\section{WILEY-VCH}

$16.01 \mathrm{eV}$ from the compressive side to the tensile side. In the center of the bent nanowire, the measured plasmon energy is about $0.1 \mathrm{eV}$ higher compared to the value measured for the straight wire. The plasmon energy in the region with the highest tensile strain matches well the experimental value measured for the unstrained nanowire.

This discrepancy between the measurement and simulation can be understood by taking the effective mass of the valence electrons into account. The bonding of the valence electrons and the electronic band structure in semiconductors can affect the plasmon energy via singleelectron excitations and, as result, shift the plasmon energy. The effect of the band structure on the plasmon resonance energy can be taken into account by replacing the electron rest mass $m_{0}$ in Equation (2) with an effective mass $m^{*} \cdot{ }^{[33]}$ Thus Equation (2) becomes

$$
E_{p}=\hbar\left[n e^{2} /\left(\varepsilon_{0} m^{*}\right)\right]^{1 / 2}=\hbar e\left(N / \varepsilon_{0}\right)^{1 / 2}\left[1 /\left(V m^{*}\right)\right]^{1 / 2} \propto\left[1 /\left(V m^{*}\right)\right]^{1 / 2}
$$

It is shown in Figure $6 \mathrm{c}$ that the experimental plasmon energy value is always higher than the value expected from the free electron model. Such a discrepancy can originate from a decrease in the effective mass of the valence electrons in the nanowire due to bending deformation. This decrease of the effective mass would counteract the effect of the volume change, which can be calculated based on geometrical considerations (Figure S4, Supporting information), on bulk plasmon energy. Such an effect is obviously more significant on the tensile side than on the compressive side (Figure 6c). In the region with the highest tensile strain, the net effect of the decrease in effective mass and the increase in lattice volume is that plasmon energy value in the tensile region is similar to that without strain. Based on the change in lattice volume and Equation (4), the amount of electron effective mass decrease was estimated to be as high as $2.5 \%$ in the tensile region of the bent area. The effective mass of electrons is directly related to the morphology of the conduction bands around the Fermi level. Hence, the change in electron effective mass shows the effect of bending deformation on the modification in the conduction band structure of the GaAs nanowires. 


\section{WILEY-VCH}

In addition to band structure variation, elastic bending deformation could give rise to other effects that can alter charge transport in nanowires. For example, strong gradients in strain due to bending deformation are likely to result in flexoelectric effects, which are normally considered insignificant in semiconductors. ${ }^{[34]}$ However, because of the 1-D morphology of the nanowire structure, much stronger strain gradients may be introduced as compared to bulk structures. In the case of the bending deformation of the GaAs nanowires, a 3\% bending strain in a nanowire with a diameter of $\sim 200 \mathrm{~nm}$ gives a strain gradient of around $3 \times 10^{5} / \mathrm{m}$ across the nanowire. Such a strong gradient could cause a considerable flexoelectric effect. The symmetry breaking between the crystal lattice structures of different regions across the nanowire would give rise to a static electric field whose field direction is perpendicular to the charge carrier transport direction. Such a field would affect the charge transport in the nanowire. Phonon scattering is another effect that might need to be taken into consideration for a complete understanding of the effect of bending strain on charge transport in nanowires. Phonon scattering is the most significant scattering mechanism at room temperature for charge carriers in semiconductors. The scattering is sensitive to the atomic arrangement and lattice structure within the material. Inhomogeneous lattice distortion caused by bending deformation could thus affect charge transport and mobility of the nanowire by the modification of phonon scattering.

In summary, a new phenomenon in the response of electrical transport properties to elastic bending deformation was observed in individual GaAs nanowires by in situ TEM measurements. The I-V characteristics of the nanowires change from linear to nonlinear as a result of the bending strain and the nonlinearity of the I-V increases with increasing strain. Contrary to the commonly anticipated band gap change due to strain, in situ STEM-EELS and SEM-CL measurements revealed that the band gap of GaAs does not change in the bent 


\section{WILEY-VCH}

region of the nanowires. Instead, the strain-induced shift of the valence band maximum can produce an energy barrier for charge carriers along the nanowire, which results in the observed change in electrical transport characteristics. Furthermore, the observed shift in the bulk plasmon peak suggests changes in the effective mass of valence electrons due to the bending deformation. The values of the effective mass of valence electrons decrease from regions under compression to regions under tension. This strong dependence of the transport properties and of the band structure of GaAs nanowires on bending deformation shows the potential of using GaAs nanowires in flexible electronics and as miniaturized mechanical sensors.

\section{Experimental section}

Nanowire growth: The p-type GaAs nanowires used in this study were grown on $\operatorname{Si}(111)$ substrate in a molecular beam epitaxy (MBE) system by a self-catalyzed vapor-liquid-solid (VLS) method. ${ }^{[35,36]}$ The growth was carried out at $630^{\circ} \mathrm{C}$ substrate temperature, with a $11 \times 10^{-6}$ Torr $\mathrm{As}_{4}$ flux, and for 45 minutes. The p-doping was achieved by adding a flux of beryllium during growth of the nanowires. The nominal carrier concentration is $\sim 3.5 \times 10^{19}$ $\mathrm{cm}^{-3}$ estimated from 4-probe electrical conductivity measurements. ${ }^{[35]}$

Sample preparation for in situ TEM: For in situ TEM measurements, single nanowires were lifted out and mounted on a TEM grid in an FEI Versa 3D FIB-SEM. We also developed a technique to attach a small piece of Au on the nanowire in FIB-SEM to improve the electrical contact on the nanowire (more details will be described in Results section). Care was taken to minimize the redeposition of Pt on the nanowire in FIB-SEM.

TEM: The in situ strain and electrical transport properties measurements were performed in a TEM using an in situ scanning tunneling microscope - transmission electron microscope 


\section{WILEY-VCH}

(STM-TEM) holder (Nanofactory AB). ${ }^{[25]}$ A TEM grid with nanowires mounted in FIB-SEM was put in the holder. The STM nanoprobe was prepared by mechanically cutting an Au wire with the diameter of $\sim 250 \mu \mathrm{m}$. The probe was used to contact the free end of the nanowire to form an electrical circuit and apply bending stress on the nanowire within the elastic regime. Measurements of the electrical transport properties of the bent nanowires were performed in TEM. A FEI Titan 80-300 TEM equipped with a probe Cs corrector, a Wien-type monochromator and a Gatan Image Filter (GIF) Tridium 866 was used for STEM imaging, EELS and in situ measurements. The microscope was operated at $300 \mathrm{kV}$. STEM annular dark field (ADF) images were taken with a beam convergence semi-angle of $\sim 17.5$ mrad. The inner and outer collection semi-angle of the ADF detector was set to be 54 and $270 \mathrm{mrad}$ respectively. The energy resolution of EELS was $\sim 70 \mathrm{meV}$ as determined by measuring the FWHM of the zero loss peak. The collection semi-angle for EELS was $\sim 5$ mrad. The induced strain was estimated based on the high magnification TEM BF images showing the geometry change of the nanowires. The electron dose in the TEM was minimized during the I-V measurements to minimize the effect of the incident electron beam on the I-V measurements. 
Supporting Information

Supporting Information is available from the Wiley Online Library.

\section{Acknowledgement}

LJZ, WJ and EO acknowledge the financial support from Swedish Research Council (VR) under Grant No. 2016-04618, Nanoscience and Nanotechnology Area of Advance and Energy Area of Advance at Chalmers University of Technology, and the European Network for Electron Microscopy (ESTEEM2, European Union Seventh Framework Programme under Grant Agreement 312483-ESTEEM2 (Integrated Infrastructure Initiative-I3)) for financial support. This project has also received funding from the European Union's Horizon 2020 research and innovation program under grant agreement No.823717-ESTEEM3. LJZ acknowledges the valuable help from Dr. Ruggero Verre in performing in situ SEM-CL measurements. TK and PK would like to acknowledge financial support by Microsoft Quantum and the European Research Council (ERC) under the grant agreement No.716655 (HEMs-DAM).

\section{References}

[1] K. Winkler, E. Bertagnolli, A. Lugstein, Nano Lett. 2015, 15, 1780.

[2] L. Sun, D. H. Kim, K. H. Oh, R. Agarwal, Nano Lett. 2013, 13, 3836.

[3] J. K. Panda, A. Roy, A. Chakraborty, I. Dasgupta, E. Hasanu, D. Ercolani, L. Sorba, M. Gemmi, Phys. Rev. B 2015, 92, 205302.

[4] D. Nam, D. S. Sukhdeo, J.-H. Kang, J. Petykiewicz, J. H. Lee, W. S. Jung, J. Vučković, M. L. Brongersma, K. C. Saraswat, Nano Lett. 2013, 13, 3118.

[5] A. M. Smith, A. M. Mohs, S. Nie, Nat. Nanotechnol. 2009, 4, 56.

[6] R. Shao, K. Zheng, B. Wei, Y. Zhang, Y. Li, X. Han, Z. Zhang, J. Zou, Nanoscale 2014, 6, 4936. 
[7] G. Signorello, E. Lörtscher, P. A. Khomyakov, S. Karg, D. L. Dheeraj, B. Gotsmann, H. Weman, H. Riel, Nat. Commun. 2014, 5, 3655.

[8] G. Signorello, S. Karg, M. T. Björk, B. Gotsmann, H. Riel, Nano Lett. 2013, 13, 917.

[9] X. Han, L. Kou, Z. Zhang, Z. Zhang, X. Zhu, J. Xu, Z. Liao, W. Guo, D. Yu, Adv. Mater. 2012, 24, 4707.

[10] Y. Chen, Q. Gao, Y. Wang, X. An, X. Liao, Y. W. Mai, H. H. Tan, J. Zou, S. P. Ringer, C. Jagadish, Nano Lett. 2015, 15, 5279.

[11] L. Zeng, C. Gammer, B. Ozdol, T. Nordqvist, J. Nygård, P. Krogstrup, A. M. Minor, W. Jäger, E. Olsson, Nano Lett. 2018, 18, 4949.

[12] S. Li, Q. Jiang, G. W. Yang, Appl. Phys. Lett. 2010, 96, 213101.

[13] R. He, P. Yang, Nat. Nanotechnol. 2006, 1, 42.

[14] X. Li, X. Wei, T. Xu, D. Pan, J. Zhao, Q. Chen, Adv. Mater. 2015, 27, 2852.

[15] K. Guilloy, N. Pauc, A. Gassenq, P. Gentile, S. Tardif, F. Rieutord, V. Calvo, Nano Lett. 2015, 15, 2429.

[16] J. Greil, S. Birner, E. Bertagnolli, A. Lugstein, Appl. Phys. Lett. 2014, 104, 163901.

[17] A. Lugstein, M. Steinmair, A. Steiger, H. Kosina, E. Bertagnolli, Nano Lett. 2010, 10, 3204.

[18] X. Fu, G. Jacopin, M. Shahmohammadi, R. Liu, M. Benameur, J.-D. Ganière, J. Feng, W. Guo, Z.-M. Liao, B. Deveaud, D. Yu, ACS Nano 2014, 8, 3412.

[19] X. Han, L. Kou, X. Lang, J. Xia, N. Wang, R. Qin, J. Lu, J. Xu, Z. Liao, X. Zhang, X. Shan, X. Song, J. Gao, W. Guo, D. Yu, Adv. Mater. 2009, 21, 4937.

[20] Y. Ahn, J. Dunning, J. Park, Nano Lett. 2005, 5, 1367.

[21] P. Krogstrup, H. I. Jørgensen, M. Heiss, O. Demichel, J. V Holm, M. Aagesen, J. Nygard, A. Fontcuberta i Morral, Nat. Photonics 2013, 7, 306.

[22] J. Dufouleur, C. Colombo, T. Garma, B. Ketterer, E. Uccelli, M. Nicotra, A. Fontcuberta i Morral, Nano Lett. 2010, 10, 1734. 
[23] H. S. Im, K. Park, J. Kim, D. Kim, J. Lee, J. A. Lee, J. Park, J. P. Ahn, ACS Omega 2018, 3, 3129.

[24] J. Wallentin, D. Jacobsson, M. Osterhoff, M. T. Borgström, T. Salditt, Nano Lett. 2017, $17,4143$.

[25] K. Svensson, Y. Jompol, H. Olin, E. Olsson, Rev. Sci. Instrum. 2003, 74, 4945.

[26] G. D. J. Smit, S. Rogge, T. M. Klapwijk, Appl. Phys. Lett. 2002, 81, 3852.

[27] J. B. Kinzel, F. J. R. Schülein, M. Weiß, L. Janker, D. D. Bühler, M. Heigl, D. Rudolph, S. Morkötter, M. Döblinger, M. Bichler, G. Abstreiter, J. J. Finley, A. Wixforth, G. Koblmüller, H. J. Krenner, ACS Nano 2016, 10, 4942.

[28] C. Thelander, P. Caroff, S. Plissard, A. W. Dey, K. A. Dick, Nano Lett. 2011, 11, 2424.

[29] M. M. McClarty, J. P. Bruce, M. S. Freund, D. R. Oliver, Appl. Phys. Lett. 2015, 106, 022107.

[30] U. Jahn, J. Lähnemann, C. Pfüller, O. Brandt, S. Breuer, B. Jenichen, M. Ramsteiner, L. Geelhaar, H. Riechert, Phys. Rev. B - Condens. Matter Mater. Phys. 2012, 85, 045323.

[31] G. Klimeck, F. Oyafuso, T. B. Boykin, R. C. Bowen, P. von Allmen, C. Comput. Model. Eng. Sci. 2002, 3, 601.

[32] R. Egerton, Electron Energy-Loss Spectroscopy in the Electron Microscope, 2011.

[33] M. H. Gass, A. J. Papworth, R. Beanland, T. J. Bullough, P. R. Chalker, Phys. Rev. B Condens. Matter Mater. Phys. 2006, 73, 035312.

[34] P. Zubko, Annu. Rev. Mater. Res. 2013, 43, DOI 10.1146/annurev-matsci-071312121634.

[35] A. Casadei, P. Krogstrup, M. Heiss, J. A. Röhr, C. Colombo, T. Ruelle, S. Upadhyay, C. B. Sorensen, J. Nygård, A. Fontcuberta i Morral, Appl. Phys. Lett. 2013, 102, 013117.

[36] P. Krogstrup, R. Popovitz-Biro, E. Johnson, M. H. Madsen, J. Nygård, H. Shtrikman, 
WILEY-VCH

Nano Lett. 2010, 10, 4475. 
Figures
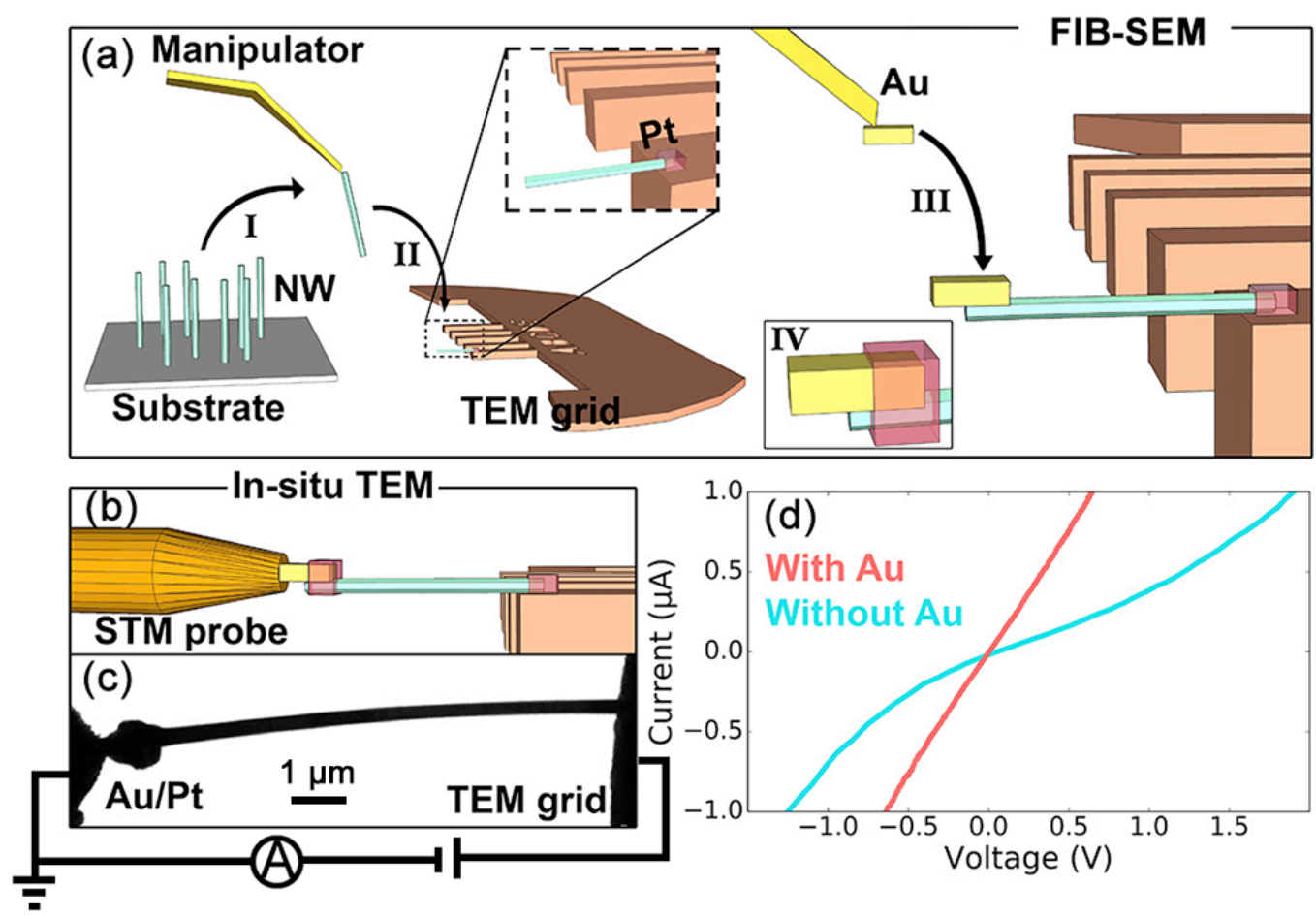

Figure 1. Method for establishing reliable electrical contacts for in situ TEM measurement. (a) Schematic diagram showing the process of transferring a GaAs nanowire onto a $\mathrm{Cu}$ TEM grid in FIB-SEM. In step I, a nanowire was lift out from the growth substrate by a micromanipulator in FIB-SEM. In step II, the nanowire was fixed on TEM grid by EBID Pt. In step III, a piece of Au metal was attached to the tip of the nanowire. Then, in step IV, the Au contact piece was welded on the nanowire with EBID Pt. (b) A sketch showing the STM nanoprobe was used to contact a $\mathrm{Au}$ piece at the tip of the nanowire to form a electric circuit in TEM. (c) A TEM BF image showing the relative position of the nanowire, Au contact at the tip of the nanowire, the STM nanoprobe and the TEM grid. (d) I-V characteristics of the nanowire with and without the Au contact piece added to the tip of the nanowire. 
(a)

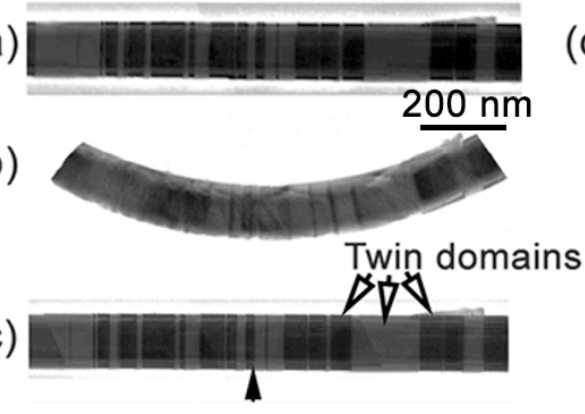

(d)

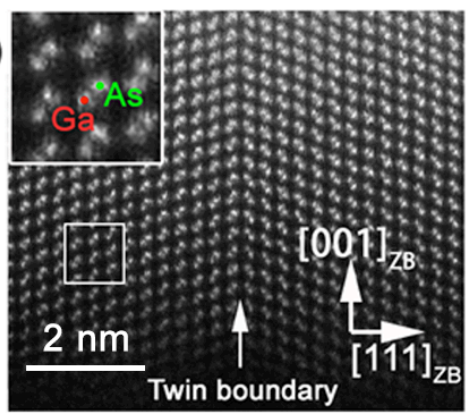

Figure 2. Microstructure of the GaAs nanowires. (a) A TEM BF image showing part of the GaAs nanowire before applying external stress. (b) A TEM BF image from the same area of the nanowire as in (a) when the nanowire was bent. (c) A TEM BF image of the nanowire when the stress was released. The alternation in image contrast along the nanowire indicates the presence of twin domains (white arrows). The black arrow indicates the position of a twin boundary that is also shown in (d). (d) A high resolution STEM ADF image showing the atomic structure of the nanowire with zinc blende structure. A twin boundary is clearly visible in the image and indicated by an arrow. The inset (top) shows the magnified images of an area marked by the white square in the main image. The positions of $\mathrm{Ga}$ and As atomic columns are marked in the inset. 


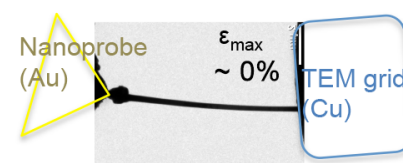

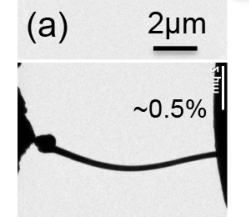

(b)

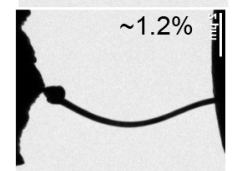

(c)

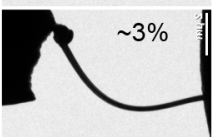

(d)
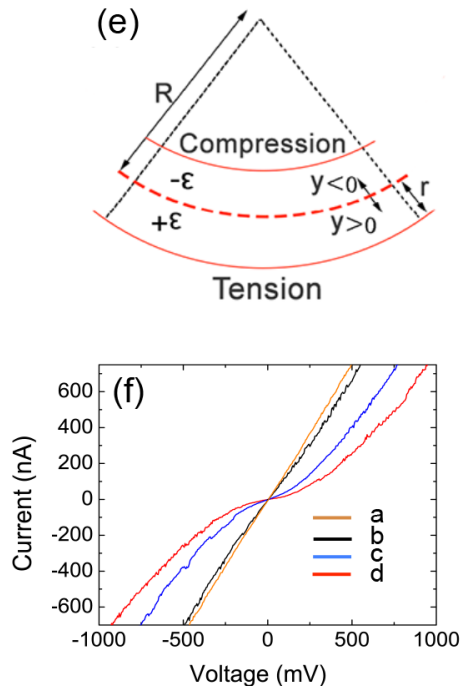

Figure 3. The effect of bending deformation on I-V characteristics of single GaAs nanowires. (a)-(d) A series of low-magnification TEM BF images showing a GaAs nanowire that was gradually bent by moving the Au nanoprobe towards the TEM grid inside the in situ TEM holder. The maximum strain $\varepsilon_{\max }$ in the nanowire is also labeled in (b)-(d). (e) Strain in the nanowire was calculated using the dimensional parameters of the bent nanowire in TEM images. $y$ is the projected distance of regions from the neutral axis of a nanowire of radius $r$, depicting strain in tension $(\varepsilon>0, y>0)$ and in compression $(\varepsilon<0, y<0) . R$ is the radius of curvature of the bent part of the wire. As a result, $\varepsilon_{\max }=r / R$. (f) I-V curves acquired at the different bending states (a)-(d) of the nanowire. 
(a)
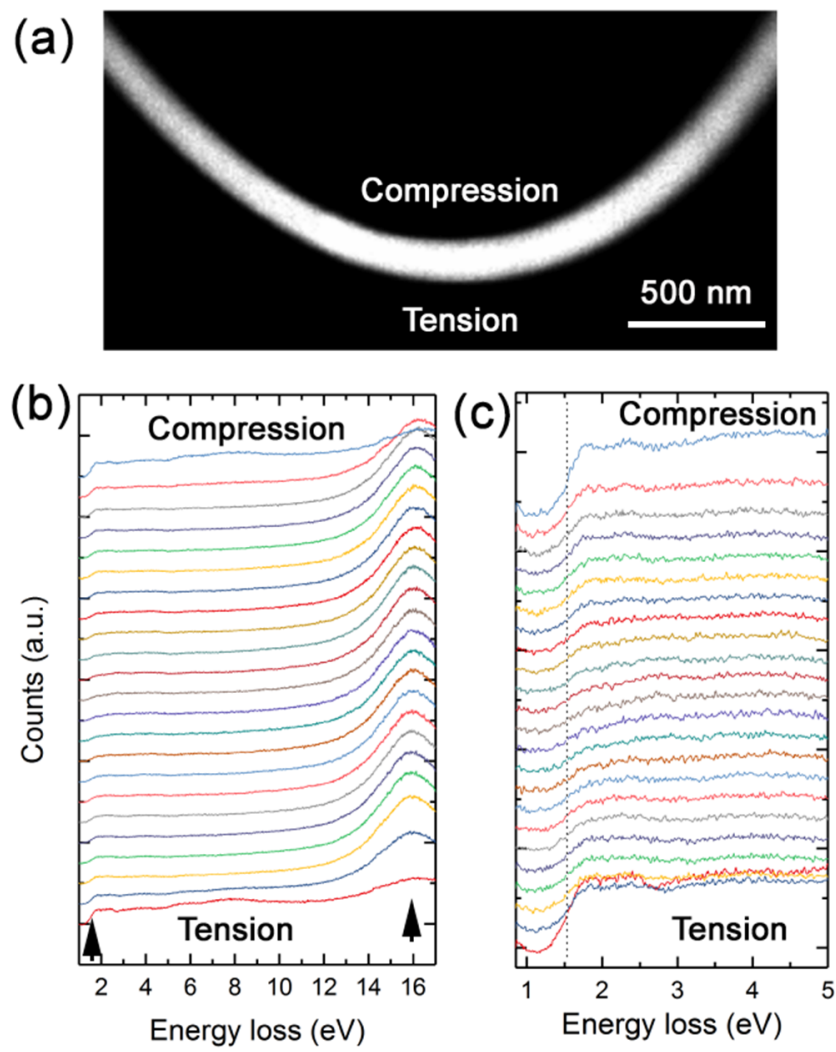

Figure 4. STEM-EELS measurement of GaAs nanowire under in situ bending deformation. (a) A STEM ADF image of a nanowire that was bent inside TEM using the in situ TEM setup. The value of $\varepsilon_{\max }$ was estimated to be $\sim 3 \%$. In the bent region, from top to bottom, the strain type in the nanowire changes from compression to tension. (b) STEM-EELS lineprofiles in the energy loss range from $\sim 1 \mathrm{eV}$ to $\sim 17 \mathrm{eV}$ taken across the GaAs nanowire under bending deformation. The arrow close to $2 \mathrm{eV}$ indicates the band gap onset position, while the arrow at around $16 \mathrm{eV}$ marks the bulk plasmon peak in the EELS spectra. (c) STEM-EELS line profile of the band gap region from compression (top) to tension side (bottom). The dotted line shows the midpoint of the band gap onset in the spectra. 


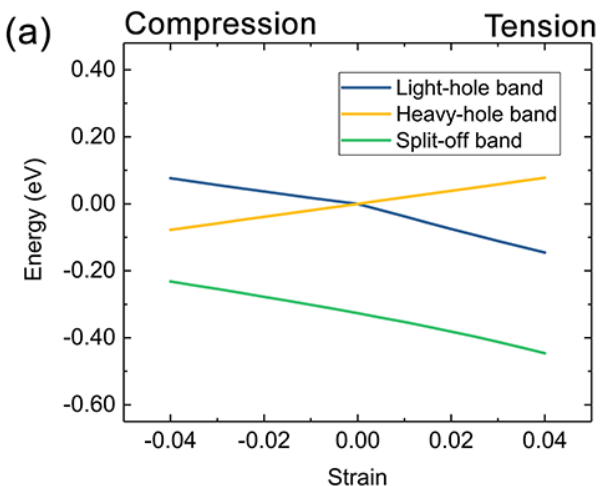

(b)

Valence band maximum

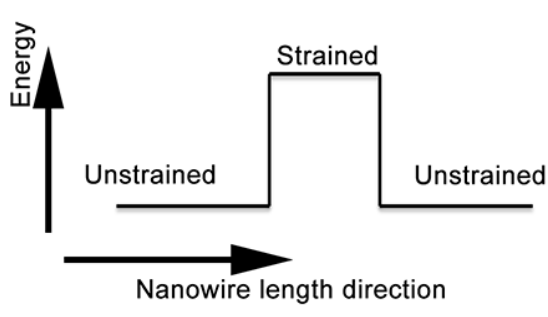

Figure 5. Shift of the valence bands due to strain: (a) $\mathrm{sp} 3 * \mathrm{~d} 5$ tight-binding simulation of the shift of the valence band top in GaAs nanowire due to strain. (b) The shift of the valence band maximum in the bent region can create an energy barrier for hole transport along the p-doped nanowire. 

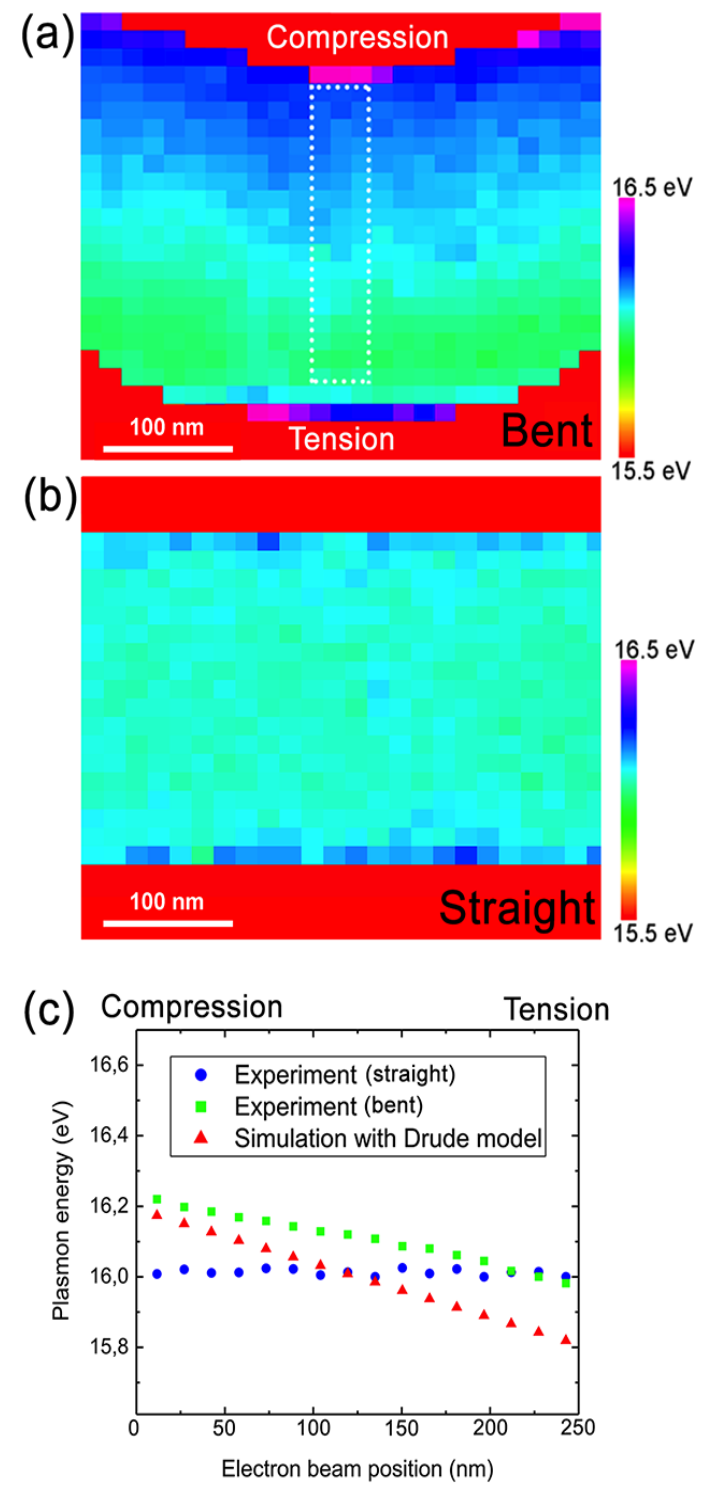

Figure 6. The effect of bending deformation on the bulk plasmon energy in GaAs nanowire. (a) Bulk plasmon energy map of a bent GaAs nanowire. (b) A map of the bulk plasmon energy from the same nanowire in (a) when it is unstrained. (c) Bulk plasmon energy as a function of electron beam position from regions of compressive strain to regions of tensile strain across the GaAs nanowire. In situ EELS measurement results are shown in green (blue) for the bent (straight) nanowire, respectively. Simulated plasmon energy as a function of beam position is also shown (red). The experimental values were extracted from the rectangular window marked in the plasmon map (a) and by averaging along the nanowire length direction. The same procedure was used to extract the plasmon energy profile for unstrained nanowire shown in (b). 


\section{WILEY-VCH}

The table of contents entry

Bending strain is used to modify the charge transport in p-doped GaAs nanowires through the development of an energy barrier for charge carriers. The sensitive response of the band structure and electrical transport property of GaAs nanowires to bending deformation shows the potential for their use in flexible electronics and sensors.

Keyword

Strain engineering

Lunjie Zeng, Thomas Kanne, Jesper Nygård, Peter Krogstrup, Wolfgang Jäger and Eva Olsson* $^{*}$

Title

The Effect of Bending Deformation on Charge Transport and Electron Effective Mass of p-doped GaAs Nanowires

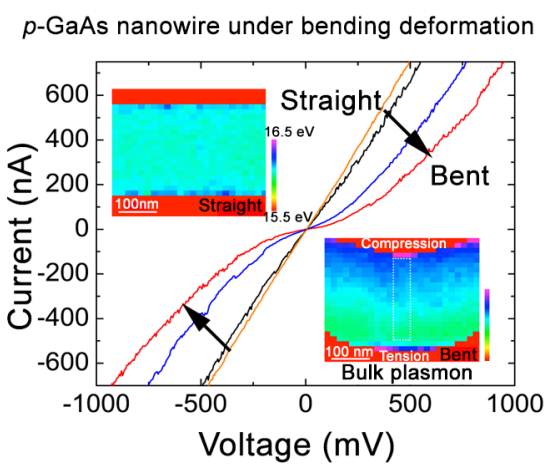


WILEY-VCH 